\title{
OPEN Photocathodes beyond NiO: charge transfer dynamics in a $\pi$-conjugated polymer functionalized with Ru photosensitizers
}

\author{
Ruri A. Wahyuono ${ }^{1,2,3}$, Bianca Seidler ${ }^{1,2,4}$, Sebastian Bold ${ }^{1,2,5}$, Andrea Dellith ${ }^{1}$, Jan Dellith ${ }^{1}$, \\ Johannes Ahner ${ }^{6,7}$, Pascal Wintergerst ${ }^{4}$, Grace Lowe ${ }^{4}$, Martin D. Hager ${ }^{6,7}$, Maria Wächtler ${ }^{1,2}$, \\ Carsten Streb ${ }^{4}$, Ulrich S. Schubert ${ }^{6,7}$, Sven Rau $^{4}$ \& Benjamin Dietzek ${ }^{1,2,7 凶}$
}

A conductive polymer (poly(p-phenylenevinylene), PPV) was covalently modified with Ru" complexes to develop an all-polymer photocathode as a conceptual alternative to dye-sensitized $\mathrm{NiO}$, which is the current state-of-the-art photocathode in solar fuels research. Photocathodes require efficient light-induced charge-transfer processes and we investigated these processes within our photocathodes using spectroscopic and spectro-electrochemical techniques. Ultrafast hole-injection dynamics in the polymer were investigated by transient absorption spectroscopy and charge transfer at the electrode-electrolyte interface was examined with chopped-light chronoamperometry. Light-induced hole injection from the photosensitizers into the PPV backbone was observed within $10 \mathrm{ps}$ and the resulting charge-separated state (CSS) recombined within $\sim 5 \mathrm{~ns}$. This is comparable to CSS lifetimes of conventional NiO-photocathodes. Chopped-light chronoamperometry indicates enhanced charge-transfer at the electrode-electrolyte interface upon sensitization of the PPV with the Ru" complexes and p-type behavior of the photocathode. The results presented here show that the polymer backbone behaves like classical molecularly sensitized NiO photocathodes and operates as a hole accepting semiconductor. This in turn demonstrates the feasibility of all-polymer photocathodes for application in solar energy conversion.

Artificial photosynthesis is a promising pathway for solar-fuel production ${ }^{1-5}$. One way of realizing an artificial photosynthetic system is to graft redox-active photosensitizers and catalysts to semiconductor electrodes to give dye-sensitized photoelectrochemical cells ${ }^{3-6}$. $\mathrm{Ru}^{\mathrm{II}}$ poly-pyridyl complexes are widely used as chromophores for dye-sensitized electrodes. They exhibit high absorptivity in the visible region and redox properties that are easily tuned by ligand modification ${ }^{1-16}$. In the case of $\mathrm{Ru}^{\mathrm{II}}$ polypyridyl complex-sensitized photocathodes for hydrogen generation, the semiconductor electrodes play the role of electron donor while the chromophores act as light harvesting and charge-separating units ${ }^{7-12}$. While metal oxide-based semiconducting electrodes such as $\mathrm{NiO}$ have attracted widespread interest ${ }^{13}$, the tuning of their physicochemical properties by chemical modification is difficult. Recently, hole conducting organic polymers have been put forward as viable alternatives for metal oxide semiconductors. This materials class has already received enormous interest as semiconducting organic components in organic photovoltaics (OPVs) ${ }^{14-16}$. A few pioneering studies have introduced such polymer-chromophore

\footnotetext{
${ }^{1}$ Department Functional Interfaces, Leibniz Institute of Photonic Technology (IPHT) Jena e.V., Albert-Einstein-Strasse 9, 07745 Jena, Germany. ${ }^{2}$ Institute of Physical Chemistry, Friedrich Schiller University Jena, Helmholtzweg 4, 07743 Jena, Germany. ${ }^{3}$ Department of Engineering Physics, Institut Teknologi Sepuluh Nopember, Jl. Arief Rahman Hakim, Surabaya 60111, Indonesia. ${ }^{4}$ Institute of Inorganic Chemistry I, Ulm University, Albert-Einstein-Allee 11, 89081 Ulm, Germany. ${ }^{5}$ Univ. Grenoble Alpes, CNRS, CEA, IRIG, Laboratoire de Chimie et Biologie des Métaux, 17 rue des Martyrs, 38000 Grenoble, France. ${ }^{6}$ Laboratory of Organic and Macromolecular Chemistry (IOMC), Friedrich Schiller University Jena, Humboldtstrasse 10, 07743 Jena, Germany. ${ }^{7}$ Center for Energy and Environmental Chemistry Jena (CEEC Jena), Friedrich Schiller University Jena, Philosophenweg 7a, 07743 Jena, Germany. ${ }^{\boxplus}$ email: benjamin.dietzek@leibniz-ipht.de
} 


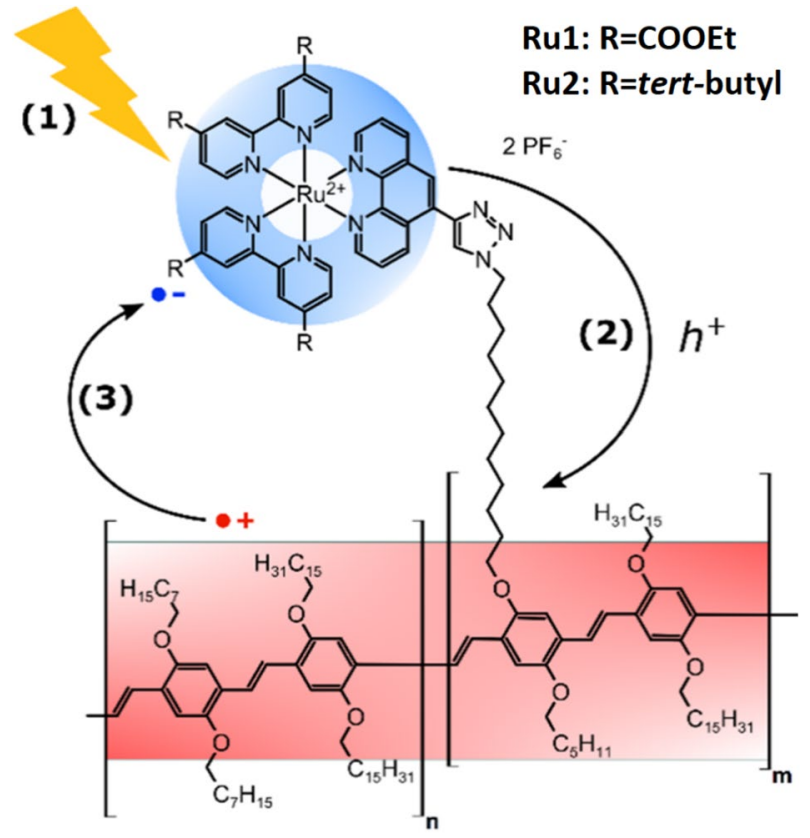

Figure 1. Schematic representation of the molecular structures of $\pi$-conjugated $\mathbf{P P V}$ with $\mathrm{Ru}^{\mathrm{II}}$ chromophores in the side chains, i.e. Ru1 and Ru2. Transient absorption spectroscopy is employed to unravel light-driven (1) hole injection (2) and charge recombination (3) dynamics in PPV-Ru. The red and blue shadings schematically indicate photogenerated holes residing on the PPV and the radical anion after hole injection from the photosensitizer to the PPV, respectively.

systems as light harvesters in artificial photosynthesis and have investigated their energy transfer properties in proof-of-principle studies ${ }^{17,18}$.

For photoinduced proton reduction Suzuki et al. ${ }^{17-20}$ developed water-insoluble polymer-photosensitizer films, in which the $\mathrm{Ru}^{\mathrm{II}}$ polypyridyl complexes were coordinated to imidazolyl residues on a partially quarternized poly(1-vinylimidazole) backbone. Schanze and co-workers ${ }^{21-23}$ reported on polystyrenes containing $\left[\mathrm{Ru}(\text { bpy })_{3}\right]^{2+}\left(\right.$ bpy $=2,2^{\prime}$-bipyridine $)$ as covalently attached pendant chromophores and $\left[\operatorname{Ru}(\right.$ tpy $)(\text { phenq) }]^{2+}$ $\left(\right.$ tpy $=2,2^{\prime} ; 6,2^{\prime \prime}$-terpyridine, phenq $=2$-(quinol- $8^{\prime}$-yl)-1,10-phenanthroline) as catalytic centers for photocatalytic water oxidation ${ }^{24,25}$. However, these studies did not exploit the electronic properties of the polymer as it acted merely as a support; charge transfer from the chromophores to a sacrificial donor and a metal oxide semiconductor anode material was required for photocatalytic proton reduction ${ }^{17-20}$ and water oxidation ${ }^{23}$, respectively. Furthermore, covalent integration of metal complexes in poly( $p$-phenylenevinylene) (PPV) derivatives has been established and the applicability of the resulting materials, e.g., in solar fuel conversion has been indicated ${ }^{26-28}$ : $\mathrm{Yu}$ and co-workers developed PPV functionalized with polypyridyl-based $\mathrm{Ru}^{\mathrm{II}}$ complexes by direct integration of the Ru-bound bipyridyl units into the $\pi$-conjugated main chain of the $\mathbf{P P V}^{26-28}$.

Inspired by Schanze's report on transition metal complexes integrated into polystyrene ${ }^{21-23}$, we propose the $\pi$-conjugated PPV as an appealing alternative hole-transport matrix for metal-oxide free photocathodes for lightdriven water splitting. Using polymers such as PPV as both a structural support and charge transport matrix utilizes the full capability of these materials. Functionalized PPV derivatives, particularly poly[2-methoxy-5-(2'ethyl-hexoxy)-1,4-phenylenevinylene] (MEH-PPV), are frequently used in polymer solar cells ${ }^{14-16,29,30}$. Importantly, their HOMO level (ca. 4.8-4.9 eV) $)^{31,32}$ is comparable to the valence band of p-type $\mathrm{NiO}$ (ca. $\left.5.0-5.1 \mathrm{eV}\right)^{33,34}$. Therefore, "molecularly sensitized PPV" could replace semiconductor electrodes in photoelectrochemical cells if the charge-transfer dynamics of the system are suitable for coupling with catalytic hydrogen evolution processes.

Herein, we report first steps towards all-polymer photocathodes for artificial photosynthesis by facile covalent attachment of Ru complexes as side-chains in PPV polymers via CLICK chemistry. The analysis of the charge transfer dynamics of these complexes reveals their suitability for visible light harvesting, charge separation and hole injection into the PPV.

\section{Results and discussion}

Synthesis of PPV-Ru. We have developed a synthetic route towards two prototype systems, PPV-Ru1 and PPV-Ru2, where $\mathrm{Ru}^{\mathrm{II}}$-polypyridyl complexes were linked covalently to the PPV backbone by CLICK chemistry (the details of the synthesis and preparation of PPV-Ru1 and PPV-Ru2 can be found in the Supplementary Information). As shown in Fig. 1, the $\mathrm{Ru}^{\mathrm{II}}$-polypyridyl complexes contain either ethyl ester (Ru1) or tertiary butyl (Ru2) substituents on their bipyridine ligands ${ }^{35}$. The photosensitizers were chosen to explore the effect of ligand substitution on the charge injection and recombination dynamics. In order to avoid the mutual interaction of two photoexcited Ru-complexes, the PPV-Ru systems investigated contained low loadings of $\mathrm{Ru}^{\mathrm{II}} \mathrm{com}$ plexes. The PPV-Ru systems contained $7.6 \%$ of either Ru1 or Ru2 related to the overall polymer backbone 


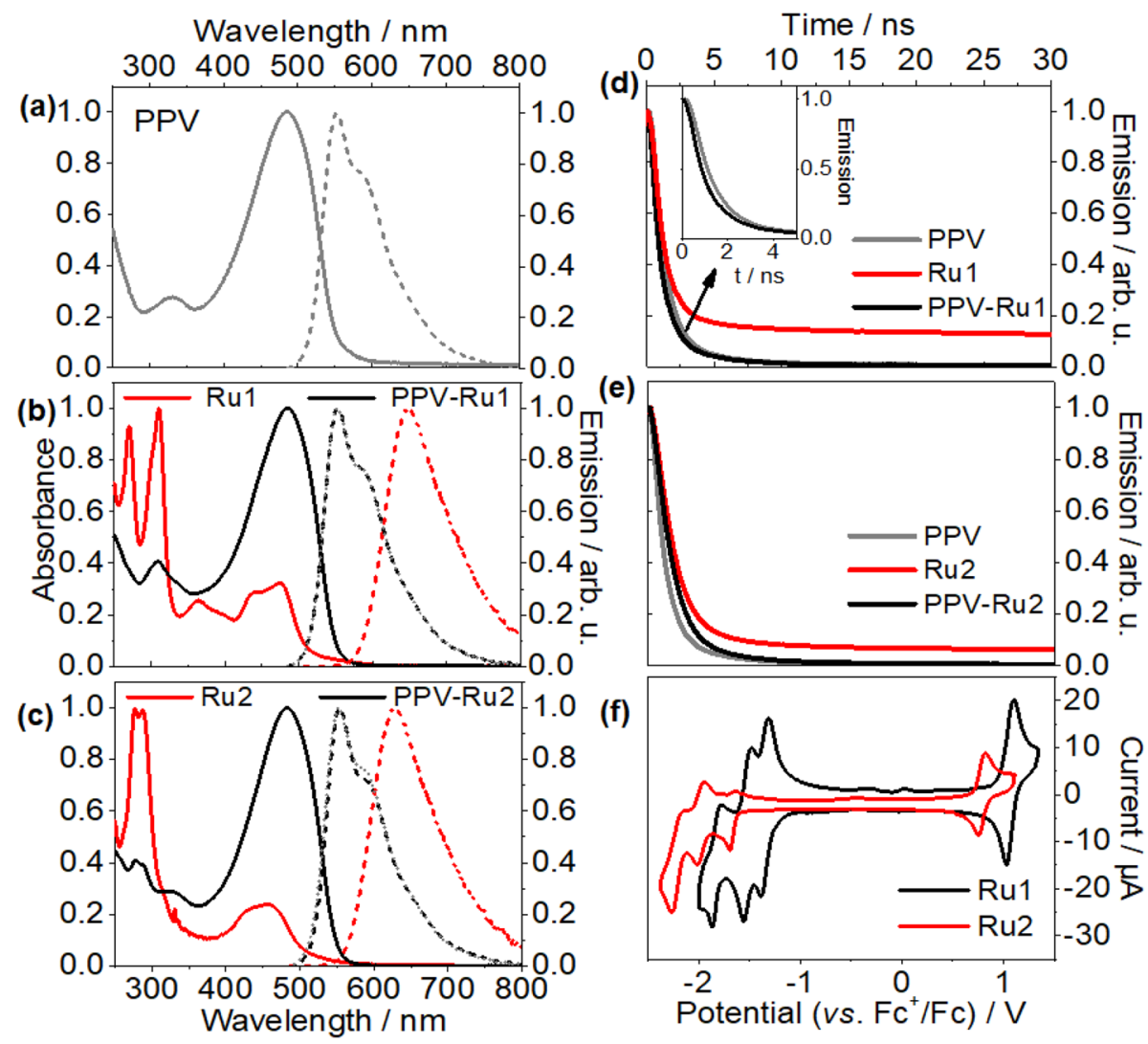

Figure 2. Normalized UV/Vis absorption and emission spectra of (a) PPV, (b) Ru1, PPV, and PPV-Ru1, and (c) Ru2, PPV, and PPV-Ru2 in $\mathrm{CHCl}_{3}$. All emission spectra were obtained upon excitation at $480 \mathrm{~nm}$. Emission decay of (d) Ru1 and PPV-Ru1, and (e) Ru2 and PPV-Ru2 in $\mathrm{CHCl}_{3}$ upon excitation at $390 \mathrm{~nm}$. The emission decay was measured using time-correlated single photon counting. The initial fast decay $(<1 \mathrm{~ns})$ arises from the instrumental response function. (f) Cyclic voltammogram of $0.25 \mathrm{mM} \mathrm{Ru} \mathbf{1}$ and $\mathbf{R u} 2$ in acetonitrile using $0.1 \mathrm{M}$ $\mathrm{TBABF}_{4}$ as supporting electrolyte.

\begin{tabular}{|c|c|c|c|c|c|c|c|c|c|c|}
\hline Structure & $\lambda_{\text {abs }}$ & $\lambda_{\mathrm{em}}\left(\Phi_{\mathrm{PL}}\right)^{\mathrm{a}}$ & $\tau_{e m}{ }^{\mathbf{b}}$ & $E_{0-0}{ }^{\mathrm{c}}$ & $E_{\text {ox }}$ & $E_{\text {red1 }}$ & $E_{\text {red2 }}$ & $E_{\text {red3 }}$ & $\Delta G_{\text {inj,e }}{ }^{\mathrm{d}}$ & $\Delta G_{\text {inj,h }}{ }^{\mathrm{e}}$ \\
\hline PPV & 485 & $553,587^{\star}(0.43)$ & $<1$ & 2.30 & +0.73 & -1.67 & - & - & - & - \\
\hline Rul & 438,476 & $646(0.06)$ & 150 & 2.17 & +1.10 & -1.33 & -1.50 & -1.79 & $0.60^{\mathrm{i}} ; 0.47^{\mathrm{ii}} ;-1.70^{\mathrm{iii}}$ & $-0.11^{\mathrm{i}} ;-0.24^{\mathrm{ii}} ;-2.41^{\mathrm{iii}}$ \\
\hline Ru2 & 430,458 & $627(0.02)$ & 139 & 2.24 & +0.82 & -1.65 & -1.96 & -2.2 & $0.25^{\mathrm{i}} ; 0.19^{\mathrm{ii}} ;-2.26^{\mathrm{iii}}$ & $0.14^{\mathrm{i}} ; 0.08^{\mathrm{ii}} ;-2.16^{\mathrm{iii}}$ \\
\hline
\end{tabular}

Table 1. Photophysical and electrochemical properties of PPV, Rul and Ru2. In nm. UV/vis absorption and emission measurements were measured in $\mathrm{CHCl}_{3}$. In $\mathrm{V}$ vs. $\mathrm{Fc}^{+} / \mathrm{Fc}$. Cyclic voltammetry was carried out at a scan rate of $100 \mathrm{mV} \cdot \mathrm{s}^{-1}$ and of $0.5 \mathrm{mM}$ complex solutions in acetonitrile containing $\mathrm{TBABF}_{4}$ as supporting electrolyte. ${ }^{a}$ Quantum yield $\left(\Phi_{\mathrm{PL}}\right)$ of $\mathbf{P P V}$ was determined using Fluorescein $(0.1 \mathrm{M} \mathrm{NaOH}$, $\left.\Phi_{\mathrm{PL}}=0.95\right)$ as reference ${ }^{46,47}$, while the quantum yields of $\mathbf{R u} \mathbf{1}$ and $\mathbf{R u} 2$ were determined using $\left[\mathrm{Ru}(\mathrm{bpy})_{3}\right]^{2+}$ (in acetonitrile, $\Phi_{\mathrm{PL}}=0.03$ ) as reference ${ }^{23}$. ${ }^{\mathrm{b}} \mathrm{In}$ ns. Emission lifetime was measured using time-correlated single photon counting. ${ }^{c} \mathrm{E}_{0-0}$ (in $\mathrm{V}$ ) is calculated from the crossing point of normalized absorption and emission spectra ${ }^{48}$. ${ }^{\mathrm{I}}$ In $\mathrm{eV}$. If only respective $\mathrm{Ru}^{\mathrm{II}}$ complex is excited: $\Delta \mathrm{G}_{\mathrm{inj,e}}=\mathrm{e}\left[\mathrm{E}\left(\mathrm{Ru}^{+/ *}\right)-\mathrm{E}\left(\mathrm{PPV}^{0 /-}\right)\right]$, where $\mathrm{E}\left(\mathrm{Ru}^{+/ *}\right)=\mathrm{E}\left(\mathrm{Ru}^{+/ 0}\right)-\mathrm{E}_{0-0}$; if only PPV is excited: $\Delta \mathrm{G}_{\text {inj,e }}=\mathrm{e}\left[\mathrm{E}\left(\mathrm{Ru}^{+/ 0}\right)-\mathrm{E}\left(\mathrm{PPV}^{* /-}\right)\right]$, where $\mathrm{E}\left(\mathrm{PPV}^{* /-}\right)=\mathrm{E}\left(\mathrm{PPV}^{0 /-}\right)+\mathrm{E}_{0-0}$; and if both PPV and Ru are excited: $\Delta \mathrm{G}_{\mathrm{inj,e}}=\mathrm{e}\left[\mathrm{E}\left(\mathrm{Ru}^{+/{ }^{*}}\right)-\mathrm{E}\left(\mathrm{PPV}^{* /-}\right)\right] .{ }^{\mathrm{e}} \mathrm{In} \mathrm{eV}$. If only Ru is excited: $\Delta \mathrm{G}_{\text {inj,h }}=\mathrm{e}\left[\mathrm{E}\left(\mathrm{PPV}^{+/ 0}\right)-\mathrm{E}\left(\mathrm{Ru}^{* /-}\right)\right]$, where $\mathrm{E}\left(\mathrm{Ru}^{* /-}\right)=\mathrm{E}\left(\mathrm{Ru}^{0 /-}\right)+\mathrm{E}_{0-0}$; if only PPV is excited: $\Delta \mathrm{G}_{\mathrm{inj}, \mathrm{h}}=\mathrm{e}\left[\mathrm{E}\left(\mathrm{PPV}^{+/ *}\right)-\mathrm{E}\left(\mathrm{Ru}^{0 /-}\right)\right]$, where $\mathrm{E}\left(\mathrm{PPV}^{+/ *}\right)=\mathrm{E}\left(\mathrm{PPV}^{+/ 0}\right)-\mathrm{E}_{0-0}$; and if both PPV and Ru are excited: $\Delta \mathrm{G}_{\mathrm{inj,h}}=\mathrm{e}\left[\mathrm{E}\left(\mathrm{PPV}^{+/ *}\right)-\mathrm{E}\left(\mathrm{Ru}^{* /-}\right)\right]$.

phenyl units. This loading corresponds to approximately one Ru-chromophore per chromophoric unit as the effective conjugation length of PPV is approximately 10 monomer units ${ }^{36-38}$.

Absorption and emission properties. UV/vis absorption and emission spectra yield first insights into the electronic structure of PPV-Ru (see Fig. 2a-c, and for summary see Table 1). The absorption spectrum of 
PPV shows an intense $\pi-\pi^{\star}$ band at $485 \mathrm{~nm}$. The $S_{1} \rightarrow S_{0}$ emission reveals a Stokes shift of $311 \mathrm{meV}^{31-34}$ and exhibits vibronic peaks at 552 and $586 \mathrm{~nm}^{39-42}$. The emission quantum yield and lifetime were determined to be $0.43 \mathrm{~ns}$ and less than $1 \mathrm{~ns}$, respectively. These values are in good agreement with previous literature reports ${ }^{39-43}$; The quantum yield is in good agreement with literature values for emission quantum yields in PPV and related systems, which fall in the range between 0.40 and $0.80^{39-42}$. The short emission lifetime is consistent with observations on, e.g., MEH-PPV and dialkoxy-substituted oligo(phenylenevinylene) ${ }^{43}$.

The UV/vis absorption spectra of $\mathbf{R u} \mathbf{1}$ and $\mathbf{R u} 2$ exhibit the characteristic features of $\mathrm{Ru}^{\mathrm{II}} \mathrm{L}_{3}$ chromophores $^{7-9,14,15,44}$. The intense absorption around $290 \mathrm{~nm}$ stems from ligand centered $\pi-\pi^{*}$ transitions localized on the bipyridine (bpy) fragments. In the visible range, Ru1 shows absorption peaks at 430 and $475 \mathrm{~nm}$ due to the absorption of metal-to-ligand charge transfer (MLCT) states. Replacing the electron-withdrawing ethyl ester (-COOEt) by electron donating tert-butyl groups blue-shifts the MLCT absorption by $100 \mathrm{meV}(18 \mathrm{~nm})$. This electronic effect of the substituent is also reflected in the more negative reduction potential of Ru2 compared with Rul (see electrochemical data below). Both photosensitizers show a weak emission with quantum yields of $0.06(\mathbf{R u 1})$ and $0.02(\mathbf{R u 2})$, which is in good agreement with the quantum yield of 0.04 observed for $\left.\mathrm{Ru}(\mathrm{bpy})_{2}(5-\mathrm{CC}-\mathrm{phen})\right]\left(\mathrm{PF}_{6}\right)_{2}{ }^{44}$.

The emission lifetime of both complexes exceeds $100 \mathrm{~ns}$ and is much longer than the emission lifetime of pristine PPV (see Table 1). Upon "sensitization" of PPV with the complexes, the UV/vis absorption and emission spectra remain similar to those of the PPV backbone, as the comparably weak MLCT absorption band of Rul and $\mathbf{R u} 2$ overlaps with the intense $\pi-\pi^{\star}$ transition of PPV. The long-lived emission component of the photosensitizer (see Fig. 2d,e) is neither observed for PPV-Ru1 nor PPV-Ru2, which might indicate quenching of the photosensitizers' long-lived emission. Literature on semiconductor-based dye-sensitized photocathodes generally associates such quenching of photosensitizer luminescence with hole transfer ${ }^{9,45}$. Thus, the apparent absence of any long-lived emission in the sensitized PPV polymers is an indication for charge separation within the polymer.

Electrochemical characterization. Electrochemical reduction/oxidation of the components allows for an estimation of the driving force for different charge transfer pathways in PPV-Ru (Fig. 2f). Due to the overlapping absorption spectra of PPV and the complexes, it is necessary to consider three possible scenarios: (i) excitation of only the Ru complexes, (ii) excitation of only the PPV backbone, and (iii) simultaneous excitation of a chromophoric unit of the PPV and a close-by photosensitizer. The estimation of the resultant driving forces for charge injection from the Ru sensitizer into the PPV based on the Gibbs free energies $(\Delta \mathrm{G})$ are summarized in Table 1. The Gibbs free energy for electron injection in scenarios (i) and (ii) is positive for both polymers, $\Delta \mathrm{G}_{\mathrm{inj}, \mathrm{e}}>0$. For PPV-Ru1 the Gibbs free energy for hole injection $\Delta \mathrm{G}_{\mathrm{inj}, \mathrm{h}}$ is negative (albeit small in magnitude), indicating that hole injection is thermodynamically possible. However, $\Delta G_{i n j, h}>0$ in PPV-Ru2. Nonetheless, considering the broad oxidation peak of PPV (Supplementary Fig. S5a), a slight negative driving force is within the expected error of the calculation, and hence, the estimated $\Delta G_{\text {inj,h }}$ for PPV-Ru2, which is close to zero, does not rule out the possibility that hole injection occurs with very little driving force. Furthermore, cathodic photocurrents for both PPV-Ru1 and PPV-Ru2 were observed in the photoelectrochemical experiments which indicates that hole injection does occur in both materials (vide infra). For scenario (iii), simultaneous excitation of $\mathrm{Ru}^{\mathrm{II}}$ complexes and $\mathbf{P P V}$, both hole and electron injection are exergonic with high injection driving forces compared to the aforementioned scenarios. Nonetheless, scenario (iii) is rather unlikely as the photon fluxes used in the presented experiments, i.e. $3.0 \times 10^{15}$ photons $\mathrm{cm}^{-2}$ pulse $\mathrm{p}^{-1}$, are only moderate. Therefore, analysis of the experimental data will consider only scenarios (i) and (ii).

Transient absorption studies of PPV-Ru in solution. The light-induced charge transfer dynamics of PPV-Ru dissolved in $\mathrm{CHCl}_{3}$ were investigated by transient absorption spectroscopy upon excitation at $480 \mathrm{~nm}$. First, transient absorption data were recorded for each individual component, PPV and each of the photosensitizers, (and this is further discussed in the Supplementary Information, see Supplementary Figs. S11-S15). Briefly, the transient absorption spectra of PPV measured in $\mathrm{CHCl}_{3}$ show an initial ground-state bleach (GSB), stimulated emission (SE) at $530 \mathrm{~nm}$ (peak) as well as $565 \mathrm{~nm}$ (shoulder), and an excited-state absorption (ESA) band beyond $600 \mathrm{~nm}$ (Fig. 3a). Quantitative analysis of the data was performed by globally fitting the data to a three-exponential decay (Table 2). This approach yields the characteristic time constants $\tau_{1}=3.8 \mathrm{ps}, \tau_{2}=72 \mathrm{ps}$, and $\tau_{3}=673 \mathrm{ps}$. The corresponding decay-associated spectra are shown in Supplementary Fig. S11c. The pumpprobe data of PPV is consistent with literature reports ${ }^{26-28,36,37}$ : The early relaxation originates from a strong coupling between electronic and vibrational states. Hence, the fast kinetic processes are attributed to delocalized exciton states (self-trapping, $100 \mathrm{fs}$ ) and vibrational cooling (few ps) ${ }^{49}$. Subsequently, the excited state dynamics is dominated by either interchain or intrachain energy transfer on a characteristic timescale of tens of $\mathrm{ps}^{50,51}$. This electronic energy transfer occurs prior to emission which generally stems from localized low-energy sites ${ }^{52,53}$.

The transient absorption spectra of both Ru1 (Fig. 3b) and Ru2 (Supplementary Fig. S13) exhibit a broad and rather unstructured ESA beyond $515 \mathrm{~nm}$ due to ligand-to-metal charge transfer transitions and a GSB centered at around $450 \mathrm{~nm}$. The ESA band at $370 \mathrm{~nm}$ is assigned to the $\pi_{1}{ }^{*} \rightarrow \pi_{2}{ }^{*}$ transition at the bpy ${ }^{--}$fragment ${ }^{9,44,46,47}$. The minor transient absorption signal decay within the experimentally accessible time window of 1.8 ns reveals the presence of a long-lived ${ }^{3}$ MLCT state typical for Ru tris-diimine complexes ${ }^{9,44}$. Quantitative analysis by global fitting with two-exponential decay functions and an infinite component (reflecting the presence of the long-lived state, see Table 2) results in characteristic time constants $\left(\tau_{1}=0.2 \mathrm{ps}, \tau_{2}=8\right.$ ps for Ru1, and $\tau_{1}=0.2 \mathrm{ps}, \tau_{2}=78 \mathrm{ps}$ for Ru2). The corresponding decay-associated spectra are shown in Supplementary Figs. S12 and S13. The pumpprobe data of the photosensitizers is consistent with literature reports on similar $\mathrm{Ru}^{\mathrm{II}}$ complexes $^{9,46,47,54-56}$ : The fast process $\left(\tau_{1}\right)$ reflects contributions from intersystem crossing (ISC, ${ }^{1} \mathrm{MLCT} \rightarrow{ }^{3} \mathrm{MLCT}$ ) and intramolecular 


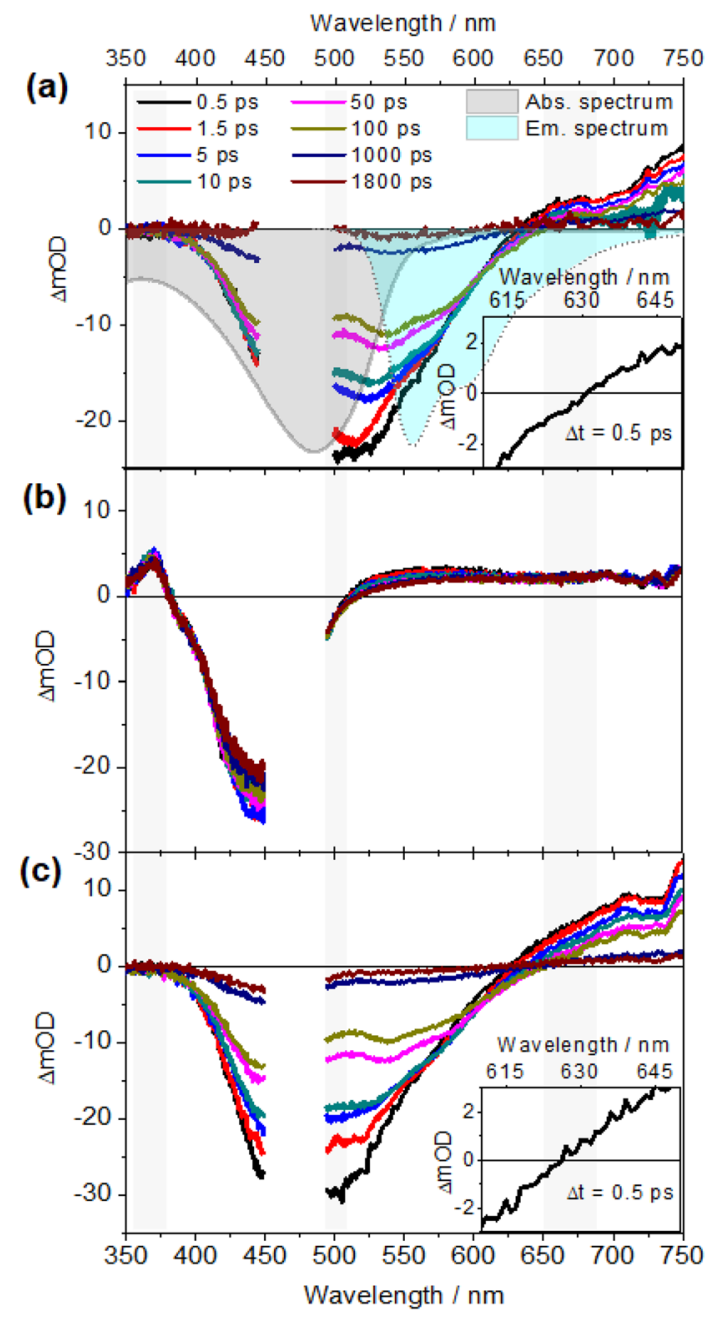

Figure 3. Fs-transient absorption spectra of (a) PPV, (b) Rul and (c) PPV-Rul at different delay times measured in $\mathrm{CHCl}_{3}$ solution with an excitation wavelength of $480 \mathrm{~nm}$. The shaded areas at 360, 500 and $670 \mathrm{~nm}$ indicate the different spectral signatures among PPV, Ru1, and PPV-Ru1. The insets in figures (a) and (c) show the zero-crossing $(\triangle \mathrm{OD}=0)$ at $0.5 \mathrm{ps}$ for $\mathbf{P P V}$ and $\mathbf{P P V}-\mathbf{R u 1}$, respectively. $\mathrm{TBABF}_{4}$ as supporting electrolyte.

\begin{tabular}{|l|l|l|l|}
\hline Structure & $\boldsymbol{\tau}_{\mathbf{1}} / \mathbf{p s}$ & $\boldsymbol{\tau}_{2} / \mathbf{p s}$ & $\boldsymbol{\tau}_{3} / \mathbf{p s}$ \\
\hline PPV & 3.8 & 72 & 673 \\
\hline Ru1 & 0.2 & 8 & Infinite \\
\hline Ru2 & 0.2 & 78 & Infinite \\
\hline
\end{tabular}

Table 2. Characteristic time constants $(\tau)$ of PPV, Ru1 and Ru2 extracted from global fit with a sum of exponential functions of TA spectra measured in solution.

vibrational redistribution. The second process $\left(\tau_{2}\right)$ corresponds to thermal relaxation within the ${ }^{3}$ MLCT manifold and leads to the population of the thermally equilibrated ${ }^{3}$ MLCT state.

Having summarized the photoinduced kinetics recorded for the individual molecular fragments, we turn our discussion to the combined system PPV-Ru. The transient absorption spectra of PPV-Ru1 (Fig. 3c) and PPVRu2 (Supplementary Fig. S15) are dominated by the spectral signature of PPV since the loading of the polymer with either of the photosensitizers is comparably low. Nonetheless, slight differences in the transient absorption spectra of PPV-Ru are observed compared to the reference PPV data; In particular the spectral shape at the red flank of the negative differential absorption band differs slightly due to spectral overlap of GSB contribution from PPV and contributions from photosensitizer-associated excited-state absorption. The zero-crossing $(\Delta \mathrm{OD}=0)$ of PPV-Ru thus shifts to a shorter wavelength at early delay time ( $\Delta t=0.5 \mathrm{ps})$ compared with PPV, i.e., from 631 to $623 \mathrm{~nm}$ for PPV-Ru1. 

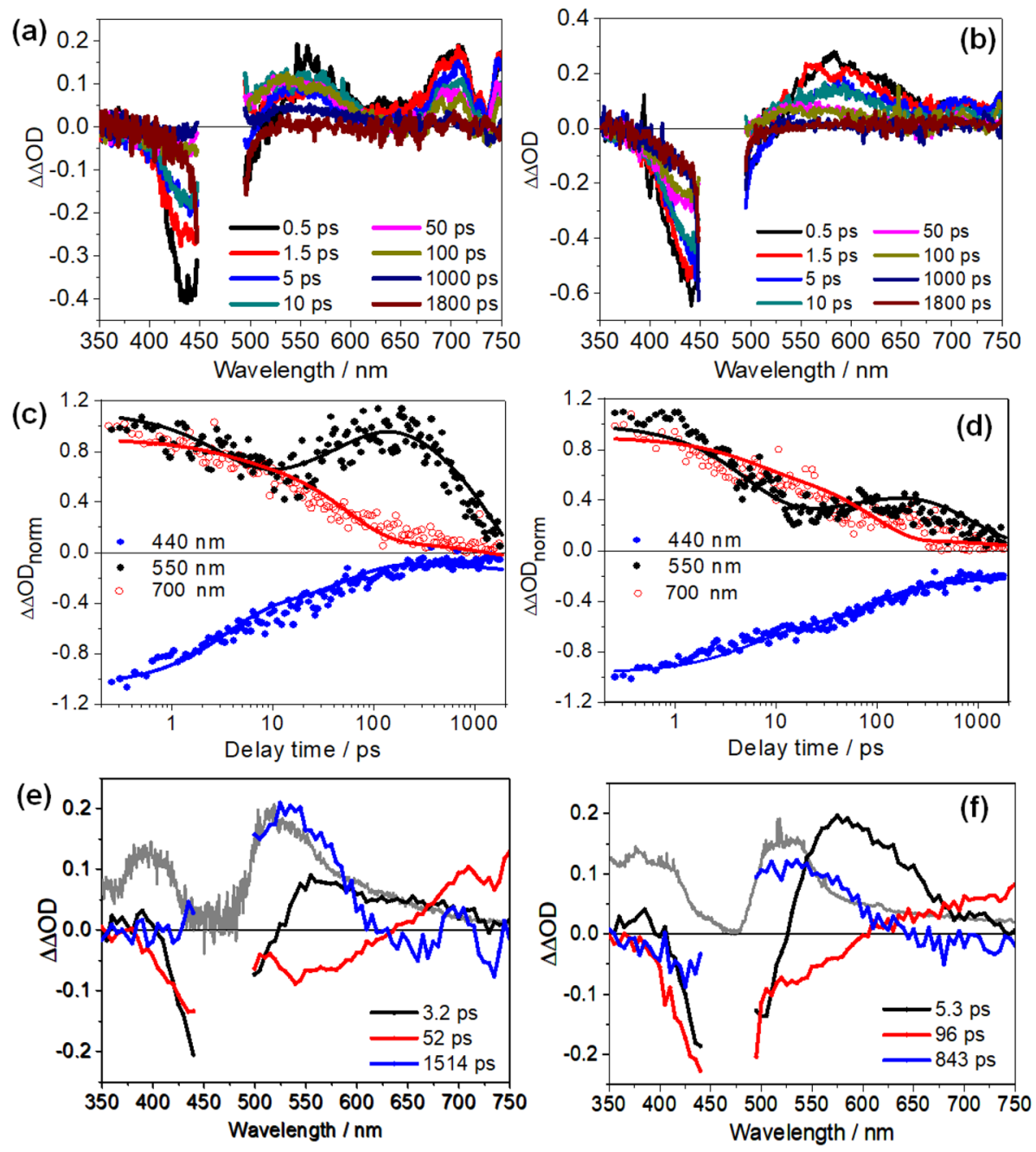

Figure 4. Differential fs-transient absorption spectra of (a) PPV-Ru1 and (b) PPV-Ru2 at different delay times. Normalized kinetic traces at different probe wavelength and decay-associated spectra (DAS) resulting from the global fit with three time constants for (c,e) PPV-Ru1 and (d,f) PPV-Ru2. The resulting time constants in the DAS are statistically taken from three independent measurements. The pump pulses are centred at $480 \mathrm{~nm}$. The grey spectra in panel (e) and (f) depict the differential absorption spectra of Ru1 and Ru2, respectively, held at a potential of slightly more negative than that of the first reduction potential (Supplementary Figs. S7 and S8), which is arbitrarily scaled to fit the transient absorption data.

The background contribution from PPV in the transient absorption spectra of the PPV-Ru systems makes the light-induced charge transfer processes difficult to analyze. To overcome this, the data obtained for PPV was subtracted from the transient absorption signal of PPV-Ru. Prior to subtraction the data is normalized to its early time $(\Delta t=0.5 \mathrm{ps})$ signal amplitude at $515 \mathrm{~nm}$. At this probe wavelength there is no contribution of the transient absorption signal from the respective $\mathbf{R} \mathbf{u}^{\mathrm{II}}$ complex (see Fig. $3 \mathrm{~b}$ ). The subtracted, i.e. differential transient absorption spectra $\left(\Delta \Delta \mathrm{OD}=\Delta \mathrm{OD}_{[\mathrm{PPV}-\mathrm{Ru}]}-\Delta \mathrm{OD}_{[\mathrm{PPV}]}\right)$, are determined as follows:

$$
\Delta \Delta O D(\Delta t, \lambda)=\frac{\Delta O D(\Delta t, \lambda)_{[P P V-R u]}}{\Delta O D(\Delta t=0.5 \mathrm{ps}, \lambda=515 \mathrm{~nm})_{[P P V-R u]}}-\frac{\Delta O D(\Delta t, \lambda)_{[P P V]}}{\Delta O D(\Delta t=0.5 \mathrm{ps}, \lambda=515 \mathrm{~nm})_{[P P V]}} .
$$

From the $\Delta \Delta \mathrm{OD}$ signal the excited state interactions between the photosensitizer and the PPV in the photoexcited PPV-Ru system can be deduced (see Fig. 4) irrespective of whether the initial excitation occurs on the PPV backbone, or in the pendant photosensitizer. The $\Delta \mathrm{OD}$ spectra recorded as a function of $\Delta t$ were globally analysed using a tri-exponential fit. The decay-associated spectra (DAS) generated from the fit are shown in Fig. $4 \mathrm{e}, \mathrm{f}$. In contrast to the dynamics of $\mathbf{R u} \mathbf{1}$ and $\mathbf{R u} 2$ in solution, the $\Delta \mathrm{OD}$ exhibits a rapid quenching of the negative signal contribution associated with GSB at $450 \mathrm{~nm}$, which occurs jointly with a decay of the positive $\Delta \Delta \mathrm{OD}$ at 560 and $700 \mathrm{~nm}$. However, from $10 \mathrm{ps}$ up to $100 \mathrm{ps}$, the $\Delta \Delta \mathrm{OD}$ signal at $550 \mathrm{~nm}$ increases again, while it continues to decrease in other spectral regions. This increase of $\Delta \Delta \mathrm{OD}(550 \mathrm{~nm})$ on a sub-100 ps timescale is followed by a slow decay of the overall signal. 
The first kinetic process characterized by $\tau_{1}, 3.3 \pm 1.2$ ps, for PPV-Ru1 and $4.0 \pm 1.3$ ps for PPV-Ru2, is assigned to energy dissipation following photoexcitation. The second process is characterized by a decay of the negative $\Delta \Delta \mathrm{OD}$ at around $450 \mathrm{~nm}$ and the built-up of positive $\Delta \Delta \mathrm{OD}$ observed at $550 \mathrm{~nm}$, which is reflected in the kinetic traces in Fig. 4c,d. This signal rise at $550 \mathrm{~nm}$ appears more prominent for Ru1, but is also observed for Ru2, and is accompanied by a decay of the positive $\Delta \Delta \mathrm{OD}$ at wavelengths longer than $610 \mathrm{~nm}$. The positive $\Delta \Delta \mathrm{OD}$ signal, which builds up with $\tau_{2}$, correlates well with the spectral change upon electrochemical reduction of either Ru1 or Ru2 (grey spectra in Fig. 4e,f). Thus, we conclude that $\tau_{2}$ is the characteristic time constant for formation of the bpy radical anion $\left(\right.$ bpy $^{-}$), i.e. $\tau_{2}$ reflects hole injection to the PPV backbone in the photoexcited PPV-Ru polymers. Hole injection generates a CSS with the hole residing in the PPV and a reduced Ru-complex. The slowest process $\left(\tau_{3}\right)$ is mainly characterized by a decay of positive $\Delta \Delta$ OD centered at $525 \mathrm{~nm}$ indicating the decay of the signal produced by the reduced ligand and hence the decay of the CSS. $\tau_{3}$ reflects the lifetime of the CSS and amounts to $994 \pm 203$ ps for PPV-Ru2 and $1584 \pm 201$ ps for PPV-Ru1. This lifetime is comparable to the reported CSS lifetime in conventional Ru polypyridyl complex-sensitized p-type semiconductors for photocatalytic hydrogen evolution ${ }^{9,46,47,54-56}$. For example, in $\mathrm{NiO}$ photocathodes sensitized with $\left[\mathrm{Ru}(\mathrm{bpy})_{3}\right]^{2+}$ based complexes, the CSS $\mathrm{h}^{+}(\mathrm{NiO}) / \mathrm{bpy}^{*-}$ is characterized by lifetimes spanning from hundreds of ps to a few ns depending on the bpy ligand substitution pattern (vide infra ${ }^{45,55-57}$.

The respective spectral changes reflecting the decay of the CSS do not involve the positive band below $425 \mathrm{~nm}$, which is observed upon electrochemical reduction of the Ru chromophores in solution (compare the blue and grey spectra plotted in Fig. 4e,f). The absence of this feature might be partially rationalized considering the strong bleach induced by the oxidation of PPV between 405 and $540 \mathrm{~nm}$ (Supplementary Fig. S5). Superposition of the this bleach with the absorption of the reduced $\mathrm{Ru}$ chromophores is also responsible for the slight shift between the spectral maxima of the $\tau_{3}$-component (blue line) and the spectrum of the reduced chromophore (grey line in Fig. 4e,f).

In principle, energy transfer from a photoexcited Ru complex to the PPV backbone to form a PPV triplet state could be a deactivation pathway working in parallel with hole injection. Similar processes have been observed in $\pi$-conjugated polymers functionalized with photosensitizers, e.g., for applications in photovoltaic and electroluminescent devices ${ }^{58-62}$. Thus, triplet energy transfer (after ultrafast intersystem crossing) from the photoexcited $\mathrm{Ru}$ sensitizers to the conjugated polymer must be considered here as well. A key observation towards increased PPV-triplet yields in PPV-Ru vs. PPV should arise from ns transient absorption ${ }^{63,64}$. However, transient absorption data recorded in the ns to $\mu$ s time range revealed no difference between PPV and PPV-Ru1 that would point to formation of PPV triplet states (Supplementary Fig. S17). Hence, we conclude that the observed spectral changes reflected in the $\Delta \Delta \mathrm{OD}$ spectra do not stem from long-lived PPV triplets, which are sensitized by the Ru chromophores, but rather from hole injection to the polymer.

Hole injection in PPV-Ru1 ( $\tau_{2}=48 \pm 4$ ps) is slightly faster than in PPV-Ru2 ( $\tau_{2}=86 \pm 9$ ps), while the CSS recombines somewhat faster in PPV-Ru2 $(994 \pm 203$ ps) than in PPV-Rul (1587 \pm 201 ps). The faster hole injection rate in PPV-Rul can be associated with the higher driving force for hole injection indicated by the more negative Gibbs free energy (see Table 1) as compared to PPV-Ru2. In addition, variations of the ligand substitution are known to alter hole injection rates in NiO-based photocathodes sensitized with $\left[\mathrm{Ru}(\mathrm{bpy})_{3}\right]^{2+}$-derived chromophores ${ }^{45,55-57}$ : e.g., $\left[\mathrm{Ru}(\mathrm{bpy})_{2}(\mathrm{dcb})\right]^{2+}\left(\mathrm{dcb}=4,4^{\prime}\right.$-dicarboxy-2,2'-bipyridine $)$-sensitized NiO does not show hole injection when anchored via the dcb ligand to the $\mathrm{NiO}$ surface. However, hole injection is observed for $\left[\mathrm{Ru}(\mathrm{dcb})_{3}\right]^{2+}$ as a sensitizer ${ }^{44}$. A related report by Dempsey and colleagues showed hole injection to NiO using $\left[\mathrm{Ru}(\mathrm{flpy})_{2}(\mathrm{dcb})\right]^{2+}\left(\right.$ flpy $=4,4^{\prime}$-bis (trifluoromethyl)-2,2'-bipyridine, dcb = 4,4'-dicarboxy-2,2'-bipyridine) as sensitizer, but not for $\left[\mathrm{Ru}(\mathrm{bpy})_{2}(\mathrm{dcb})\right]^{2+}$. The authors associated their result with the stronger electron withdrawing character of the utilized $-\mathrm{CF}_{3}$ on bpy-ancillary ligands ${ }^{56}$. Considering sensitized metal-oxide photocathodes such effects of ligand-substitution and related findings associated with the lifetime of the $\mathrm{CSS}^{4}$ can be rationalized by taking into account a fixed orientation of the photosensitizer (and hence the ligand-centered radical anion) with respect to the semiconductor. Such arguments are not straightforward in PPV-Ru, as hole transfer will occur through space and it is not a priori evident if the closest distance between the photosensitizer and a chromophoric unit of the (generally) coiled PPV is via the clicked phenanthroline or the bipyridine ligands. Nonetheless, the experimental data provides evidence for sensitive structural control of the charge injection dynamics via liganddesign when considering hole injection, not only to $\mathrm{NiO}$ but also into potential soft-matter electrodes.

Transient absorption studies of PPV-Ru in films. To evaluate the potential of PPV-Ru as materials for all-polymer photoelectrodes, we studied the photoinduced charge-transfer processes in drop-cast films of PPV-Ru1 (see Fig. 5). Films were prepared by drop-casting a solution of PPV-Ru1 in $\mathrm{CH}_{3} \mathrm{Cl}(10 \%$ w/v) onto PET/ITO substrates. This gave relatively smooth films with a thickness of $300 \pm 16 \mathrm{~nm}$, which is in the lower range of typical thicknesses for $\mathrm{NiO}$-based photocathodes ${ }^{9,10,45}$. Following the same data analysis as laid out above, $\Delta \Delta$ OD spectra are obtained for PPV-Ru1 (Fig. 5b). The $\Delta \Delta$ OD shows negative features between 400 and $580 \mathrm{~nm}$, which are broader than for PPV-Ru1 in solution. Furthermore, the $\Delta \Delta$ OD kinetics reveal differences between PPV-Ru1 in film and in solution (Fig. 5c). For PPV-Rul films, the $\Delta \Delta \mathrm{OD}$ features a rapid initial decay of the ground state bleach at $530 \mathrm{~nm}$ and a positive $\Delta \Delta \mathrm{OD}$ band at $740 \mathrm{~nm}$. The $\Delta \Delta O D$ signal at $600 \mathrm{~nm}$ increases on a timescale of up to $20 \mathrm{ps}$ and is followed by an overall decay of the signal.

The $\Delta \Delta$ OD data of PPV-Ru1 is analysed using a tri-exponential fit (see Fig. 5d). The first decay component characterized by $\tau_{1}=8.3 \pm 2.6 \mathrm{ps}$ reflects a build-up of positive $\Delta \Delta \mathrm{OD}$ at around $600 \mathrm{~nm}$ accompanied by a loss in ground-state bleach. The signal build-up at $600 \mathrm{~nm}$ can be correlated with the spectral changes upon electrochemical reduction of Ru1 in PPV-Ru1 (grey spectrum in Fig. 5d). However, the positive DAS $\left(\tau_{2,3}\right)$ feature observed above $600 \mathrm{~nm}$ extends further to the blue than the signal reflecting the reduction of the Ruchromophore in PPV-Ru1. This red shift could be due to the contribution of a broader GSB feature of Rul 

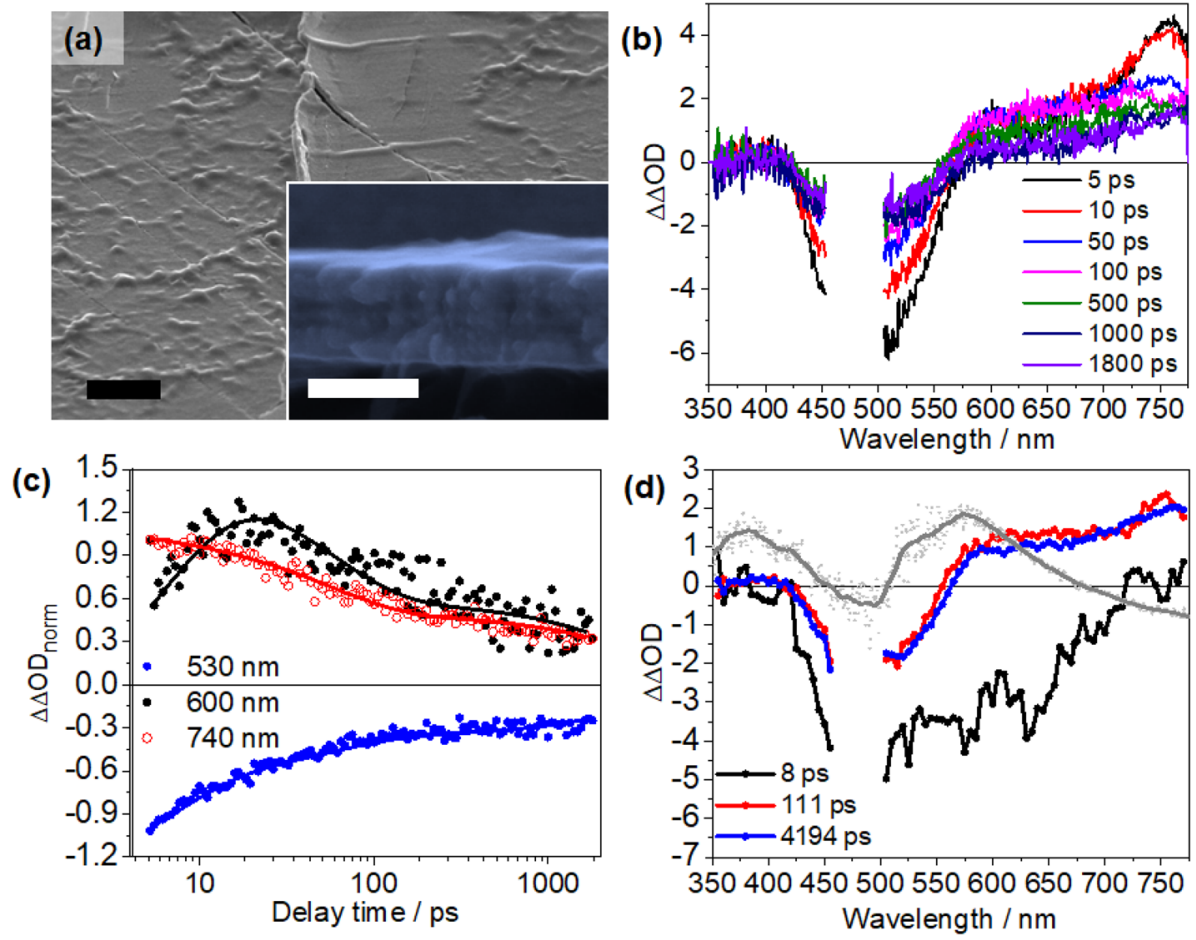

Figure 5. (a) Micromorphology of a PPV-Rul film dropcasted onto PET/ITO substrate. The inset depicts the cross-section image of PPV-Ru1 photocathodes. The black and white scale bar in panel (a) indicate $3 \mu \mathrm{m}$ and $300 \mathrm{~nm}$, respectively. (b) Fs-transient absorption spectra, (c) normalized kinetic traces recorded at different probe wavelength, and (d) decay-associated spectra (DAS) resulting from the global fit of the transient absorption data using a tri-exponential model. The grey spectra in panel (d) depict the absorption-difference spectra upon chemical reduction of Rul (at - $1.4 \mathrm{~V}$ vs. Ag|AgCl) in the PPV-Rul film. The absorptiondifference spectra (Supplementary Fig. S10) are arbitrarily scaled to the figure.

measured in film which 410 and $575 \mathrm{~nm}$ (see Supplementary Fig. S19). Therefore, $\tau_{1}$ is assigned to hole injection. The subsequent processes associated with $\tau_{2}$ and $\tau_{3}$ are characterized by a decay of the reduced Rul, i.e. the positive $\Delta \Delta \mathrm{OD}$ band $>550 \mathrm{~nm}$. Charge recombination, i.e. full decay of the CSSs due to recombination occurs bi-exponentially with $\tau_{2}=107 \pm 19 \mathrm{ps}$ and the significantly longer $\tau_{3}=4.8 \pm 1.1 \mathrm{~ns}$. Hence, it is apparent that the rate of hole injection from the photosensitizer to the polymer backbone and (partial) charge recombination in PPV-Rul are faster in the film than in solution.

We propose that the difference in the hole-injection rates for the solution phase and drop-caste polymer can be rationalized by considering "frozen geometries" in the polymer film; In these 'geometries' a sensitizer and a chromophoric unit of the PPV are arranged favourably for electronic coupling. As a result, hole injection and charge recombination occur more rapidly in the drop-caste polymer than for the polymer in solution. In solution the polymer can fluctuate in and out of such "optimal" geometries. These slow structural fluctuations available to the polymer in solution allow the system to sample a large conformational space, which ultimately leads to charge recombination with a characteristic time-constant of roughly $1 \mathrm{~ns}$. In the PPV-Ru1 films sample, the hole injected into the PPV backbone upon creation of the CSS initially resides close to the $\mathrm{Ru}^{\mathrm{II}}$ complex. It can then be transferred to other chromophoric units of the polymer which were not involved in the initial hole injection process. Upon such hole migration ${ }^{65,66}$, the hole residing in the PPV becomes spatially separated from the reduced $\mathrm{Ru}$ chromophore and hence charge recombination becomes hindered. Depending on the actual substitution pattern of PPV hole migration occurs as fast as within $100 \mathrm{ps}^{65,66}$. For BEH-PPV, i.e. poly[2,5bis(2'-ethyl-hexyl)-1,4-phenylenevinylene, a substitution pattern similar to the one used here, hole characteristic migration times of $180 \mathrm{ps}$ have been determined in a pristine thin film ${ }^{67,68}$. Thus, hole migration occurs on a time-scale comparable to the decay of the primary CSS in PPV-Rul films. The presence of two distinct charge recombination channels in PPV-Rul films is thus attributed to the presence of two different ensembles of CSS in the polymer: one with optimal electronic coupling between the hole and the reduced chromophore (responsible for the comparably fast decay characterized by $\tau_{2}$ ), and another set of geometries in which the hole has been further separated from the reduced $\mathrm{Ru}^{\mathrm{II}}$ photosensitizer by hole transfer in the polymer (responsible for the slow charge recombination characterized by $\tau_{3}$ ).

Photoelectrochemical measurements. The charge-transfer behavior of the photoexcited polymers was investigated using linear scan voltammetry (LSV) and chronoamperometry under chopped irradiation (white light, $1000 \mathrm{~W} \mathrm{~m}^{-2}$ ). The experiments were carried out on drop-caste films of the polymers using a three-electrode spectroelectrochemical cell and a $\mathrm{Co}^{\mathrm{III}} / \mathrm{Co}^{\mathrm{II}}$ redox couple as a sacrificial electron acceptor (Fig. 6). 

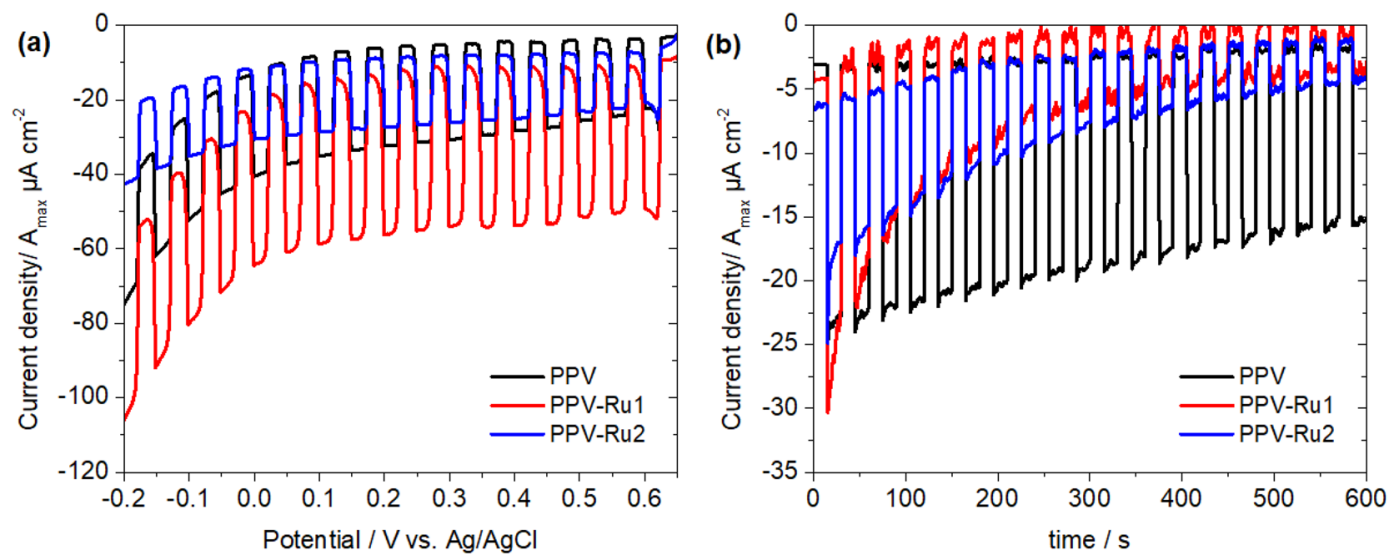

Figure 6. Results of the photoelectrochemical measurements performed on drop-casted films of PPV, PPV-Ru1 and PPV-Ru2 in $\mathrm{Co}^{\mathrm{III}} / \mathrm{Co}^{\mathrm{II}}$ electrolyte under chopped illumination $(1000 \mathrm{~nm}>\lambda>300 \mathrm{~nm}$, $1000 \mathrm{~W} \cdot \mathrm{m}^{-2}$ ). (a) Linear scan voltammogram (scan speed $20 \mathrm{mV} / \mathrm{s}$ ) and (b) chronoamperometric measurement at $0.5 \mathrm{~V}$ vs. $\mathrm{Ag} / \mathrm{AgCl}$ applied potential. The obtained currents were divided by the film surface area to obtain current densities, and further divided by the maximum absorbance $\left(\mathrm{A}_{\max }\right)$ of the films to account for film thickness. Shown are the average values of four individual measurements.

In the LSV experiments, all polymers show a cathodic photocurrent that is independent of the applied potential, while the dark current increases steeply below ca. 0 V vs. Ag/AgCl. Of the three polymers, PPV-Ru1 shows the highest photocurrent with $42 \mu \mathrm{A} \mathrm{cm}^{-2} \mathrm{~A}_{\max }{ }^{-1}\left(6.5 \mu \mathrm{A} \mathrm{cm}^{-2}\right.$ current density before normalization to the absorbance, see Supplementary Fig. S22), which is in line with the faster hole injection and slower charge recombination observed in the TA experiments. Compared to the conventional $\left[\mathrm{Ru}(\mathrm{bpy})_{3}\right]^{2+}$-sensitized NiO photocathode, the resulting current density in PPV-Rul is at this stage of the proof-of-concept study notably lower $\left(6.5 \mathrm{vs} 120 \mu \mathrm{A} \mathrm{cm}^{-2}\right)^{9}$. Nonetheless, this current density is found on par to the organic push-pull dye-sensitized $\mathrm{NiO}$ photocathodes which yield photocurrent density in the range of $2.2-6 \mu \mathrm{A} \mathrm{cm} \mathrm{cm}^{-269,70}$.

In the chronoamperometric experiments, PPV-Rul also initially shows the highest photocurrent density. However, this decreases by $90 \%$ during the duration of the experiment ( $10 \mathrm{~min})$. While PPV-Ru2 shows a similar behavior, the non-functionalized PPV film shows a much slower decrease in photocurrent density $(35 \%$ loss after $10 \mathrm{~min}$ ). The decrease in photo-current density during the chopped-light measurements indicates that the investigated polymers are rather unstable under irradiation, which is supported by the strong decrease in absorbance of the PPV absorption band in post-operando UV-Vis spectra of the films (see Supplementary Fig. S23). Therefore, this instability is likely due to the poor photostability of the PPV backbone itself ${ }^{67,68}$. We ascribe the acceleration of the decay in PPV-Rul to the prolonged charge-separated state lifetime in comparison with pristine PPV, which could lead to faster degradation of the PPV backbone in it's oxidized state. The extension of the charge-separated state lifetime is therefore a double-edged sword, leading to both an increase in activity and a decrease in stability. However, because of the modular nature of these soft matter materials, in future work both the polymer backbone and sensitizer components can be synthetically optimized to produce more stable soft-matter photocathodes.

\section{Conclusions}

We have demonstrated the photosensitization of PPV with different polypyridyl $\mathrm{Ru}^{\mathrm{II}}$ complexes through CLICK chemistry. The charge transfer dynamics in the polymer were unravelled using time-resolved optical spectroscopy. In both polymers a CSS was formed by light-driven hole injection from the photosensitizer into the PPV backbone. While photoinduced hole transfer in drop-cast films of PPV-Ru1 took place in less than 10 ps, it was found to take place on a sub-100 ps timescale for samples in solution. The CSS lifetimes in solution and in drop-caste films were comparable to that observed in very recent studies ${ }^{45,55-57}$ for conventional NiO-based photocathodes. Altering the ligands of the $\mathrm{Ru}^{\mathrm{II}}$ complexes modified hole injection and recombination rates within the sensitized polymers. An electron-withdrawing group on the bipyridyl ligand gave rise to faster hole injection into the polymer backbone and slower charge recombination. As a proof-of-concept, we showed that the PPV-Ru system works as a functional photocathode indicated by the generation of a cathodic current in a photoelectrochemical experiment. Thus, we have shown that light induced charge transfer occurs between PPV and ruthenium complexes attached to the polymer via side chains. This leads to sufficiently long-lived charge separated states to allow electron transfer to acceptor species in solution. Furthermore, we have demonstrated a modular design approach implementing CLICK chemistry at innocent phenanthroline ligands to attach two different ruthenium complexes (Ru1 and Ru2 with chemically very different ligand substitution patterns). This indicates that sophisticated supramolecular photocatalyst architectures can be introduced ${ }^{69}$, permitting the implementation of well-established synthetic chemistry for tris-heteroleptic ruthenium building blocks ${ }^{70}$. 
We intend to further exploit the concept introduced here to develop "molecularly functionalized" hole-conducting polymer photocathodes as an alternative to conventional $\mathrm{NiO}$ photoelectrodes. Ultimately, this research should lead to the realization of soft-matter photoelectrochemical cells for solar fuel generation.

\section{Experimental section}

Synthesis and preparation of PPV, Ru's, and PPV-Ru's. Detail of synthetic procedure of PPV, Ru's, and PPV-Ru's including materials and instrumentation is described in the Supplementary Information.

Spectroscopy and (Spectro)electrochemical measurements. UV/vis absorption and emission measurement. UV/vis absorption and emission spectra of PPV, Ru1, Ru2, PPV-Ru1 and PPV-Ru2 were measured in $\mathrm{CHCl}_{3}$ using a Varian-Cary UV-Vis-NIR spectrometer and FLS980 photoluminescence spectrometer (Edinburg Instrument), respectively ${ }^{71}$.

Spectroelectrochemical measurements. Each solution contained $0.1 \mathrm{M}$ tetrabutylammonium tetrafluoroborate (Sigma-Aldrich, for electrochemical analysis, $\geq 99.0 \%$ ) in $\mathrm{CH}_{3} \mathrm{Cl}$ as supporting electrolyte. The setup for both electrochemical and spectroelectrochemical measurements was described before ${ }^{72,73}$ : A thin-layer $1 \mathrm{~mm}$ path length quartz glass spectroelectrochemical cell (Bioanalytical Systems, Inc.), equipped with a Pt counter electrode, a Ag|AgCl-pseudoreference electrode and a glassy carbon working electrode with a slit of $2 \mathrm{~mm} \times 5 \mathrm{~mm}$, was used. The redox potentials were determined by cyclic voltammetry using a VersaSTAT 3 potentiostat (Princeton Applied Research). UV/Vis spectra were collected during reduction of the complexes, i.e. during chronoamperometry at various potentials. The UV/Vis spectra were obtained using a single channel fiber optic spectrometer (Avantes Inc., AvaSpec-ULS2048XL) with a deuterium-halogen light source (Avantes Inc., AvaLight DH-S-BAL). For UV/vis spectroelectrochemistry of PPV-Ru1 films, $20 \mu \mathrm{L}$ of PPV-Ru1 solution (either $10 \%$ or $20 \% \mathrm{w} / \mathrm{v}$ ) in $\mathrm{CH}_{3} \mathrm{Cl}$ was drop-caste on transparent PET/ITO (Indium-doped Tin Oxide) substrate. The PPV-Ru1 film thickness was determined from the cross-section images obtained by using JEOL JSM6300F scanning electron microscope operating at $5.0 \mathrm{kV}$ accelerating voltage. The UV/vis spectra were obtained in transmission mode through the PET/ITO/PPV-Rul working electrode using the same spectrometer and light source as mentioned above.

Time-resolved emission measurements. Time-resolved emission measurements were carried out by using timecorrelated single-photon counting (TCSPC) ${ }^{45}$. A Ti:Sapphire laser (Tsunami, Newport Spectra-Physics GmbH) was used as the light source. The repetition rate is reduced to $400 \mathrm{kHz}$ by a pulse selector (model 3980, Newport Spectra-Physics GmbH). Afterwards, the fundamental beam of the Ti-Sapphire oscillator is frequency doubled in a second harmonic generator (Newport Spectra-Physics GmbH) to create the 390-nm pump beam. The emission was detected by a Becker and Hickel PMC-100-4 photon-counting module. The emission lifetimes were determined by fitting a monoexponential decay to the data.

Transient absorption measurements. The setup for femtosecond (fs) - transient absorption measurements of PPV, Ru1, Ru2, PPV-Ru1, and PPV-Ru2 both in solutions and on films was previously described ${ }^{74-76}$ : The $800 \mathrm{~nm}$ output of an amplified Ti:Sapphire laser (Legend, Coherent Inc.) was split into two beams, one of which was used to pump an optical-parametric amplifier (TOPAS-C). The TOPAS output pulses were spectrally centered at $480 \mathrm{~nm}$ with a pump-pulse energy of typically $1.2 \mu \mathrm{J}$ and used as pump pulses in the fs pump-probe experiments. This pump beam was directed over a $600 \mathrm{~mm}$ delay line in order to realize the temporal delay of up to $\sim 2$ ns between the pump and the probe pulses. The residual fraction of the fundamental was employed for supercontinuum generation in $\mathrm{a} \mathrm{CaF}_{2}$ window used as a broad-band probe in the transient absorption experiments, in which the typical probe intensities fall into the range of hundred $\mathrm{nJ}$. The probe light was then split into two beams, one of which was focused into the sample by means of a $500 \mathrm{~mm}$ focal length spherical mirror, while the second beam was used as reference. The mutual polarizations of pump and probe were set to magic angle. Probe and reference intensities were detected on a double-stripe diode array and converted into differential absorption (DA) signals using a commercially available detection system (Pascher Instruments AB, Sweden). Chirp correction and subsequently a global fit routine using a sum of exponentials was carried out for data analysis $^{75,76}$. To avoid prominent contributions from coherent artifacts ${ }^{77,78}$, the pulse overlap region $( \pm 250 \mathrm{fs}$ around time zero) was excluded from the data fitting procedure. For solution measurement, samples were dissolved in $\mathrm{CHCl}_{3}$ in a quartz cell with $1 \mathrm{~mm}$ optical path length, while the PPV, Ru1, Ru2, PPV-Ru1, and PPVRu2 film were prepared by drop casting $100 \mu \mathrm{L}$ of solution $(10 \% \mathrm{w} / \mathrm{v})$ on ITO coated microscope slide. Both solution and film samples were moved during scans to allow the pump-probe beam to hit the fresh sample area.

The nanosecond (ns) transient measurements were carried out to investigate the long-lived species in PPV and PPV-Ru in solutions upon photoexcitation. All measurements were undertaken in $1 \mathrm{~cm}$ path length fluorescence cuvettes. The setup for ns-transient absorption measurements was previously described ${ }^{79,80}$ : The pump pulse was centered at $480 \mathrm{~nm}$ and generated by a Continuum Surelite OPO Plus apparatus (pumped by an Nd:YAG laser system, pulse duration $5 \mathrm{~ns}$, repetition rate $10 \mathrm{~Hz}$ ). A $75 \mathrm{~W}$ xenon arc lamp was used as the probe light. Spherical concave mirrors were utilized to focus the probe light into the samples as well as to the monochromator (Acton, Princeton Instruments). The probe light was detected by a Hamamatsu R928 photomultiplier. The signal was amplified and processed by a commercially available detection system (Pascher Instruments $A B$ ). For all measurements, the pump pulse energy was kept at $0.4 \mathrm{~mJ}$. A spectral band of $20 \mathrm{~nm}$ around the pump wavelength is omitted from the data analysis due to pump-scatter in this spectral range. 
Photoelectrochemical measurements. Photoelectrochemical measurements were conducted in a threeelectrode cell. The working electrode was a polymer-masked (Surlyn) PET/ITO substrate which was coated with the PPV(-Ru) polymer films by drop-casting: $100 \mu \mathrm{L}$ of polymer solution $(1 \mathrm{mg} / \mathrm{mL})$ in $\mathrm{CHCl}_{3}$ was drop-casted on a masked PET/ITO substrate of ca. $1 \times 3 \mathrm{~cm}$ size. After evaporation of the solvent the mask was removed to yield substrates of ca. $1 \times 1 \mathrm{~cm}$ size. The reference electrode was a leakless $\mathrm{Ag} \mid \mathrm{AgCl}$ electrode (EDAQ) and the counter electrode was a $\mathrm{Pt}$ wire. $\mathrm{A} \mathrm{Co}{ }^{\mathrm{II}} / \mathrm{Co}^{\mathrm{II}}$ redox couple $(0.25 \mathrm{M}$ tris(2-(1H-pyrazol-1-yl) pyridine) cobalt(II) di[bis(trifluoromethane)sulfonimide, $0.06 \mathrm{M}$ tris(2-(1H-pyrazol-1-yl)pyridine)cobalt(III) tri[bis(trifluoromethane)sulfonimide), was added to the supporting electrolyte solution (0.1 M LiTFSI, and $0.5 \mathrm{M}$ 4-tert-butyl pyridine in dry acetonitrile). Linear scan voltammograms were recorded at $20 \mathrm{mV} / \mathrm{s} \mathrm{scan}$ speed with chopped light illumination $\left(1000 \mathrm{~nm}>\lambda>300 \mathrm{~nm}, 1000 \mathrm{~W} \mathrm{~m}^{-2}, 5 \mathrm{~s}\right.$ cycle time) provided by a light source connected to a Zahner potentiostat (Zennium Pro). The chronoamperometric measurements were performed with an applied potential of $0.5 \mathrm{~V}$ vs. $\mathrm{Ag} \mid \mathrm{AgCl}$ and using the same light source with a cycle time of $30 \mathrm{~s}$.

Received: 3 September 2020; Accepted: 19 January 2021

Published online: 02 February 2021

\section{References}

1. Thapper, A. et al. Artificial photosynthesis for solar fuels-An evolving research fields within AMPEA, a joint programme of the European energy research alliance. Green. 3, 43 (2013).

2. Berardi, S. et al. Molecular artificial photosynthesis. Chem. Soc. Rev. 43, 7501 (2014).

3. Black, F. A. et al. Charge-transfer dynamics at the dye-semiconductor interface of photocathodes for solar energy applications. Faraday Discuss. 198, 449 (2017)

4. Brennaman, M. K. et al. Finding the way to solar fuels with dye-sensitized photoelectrosynthesis cells. J. Am. Chem. Soc. 138, 13085 (2016).

5. Andreiadis, E. S., Chavarot-Kerlidou, M., Fontecave, M. \& Artero, V. Artificial photosynthesis: From molecular catalysts for lightdriven water splitting to photoelectrochemical cells. Photochem. Photobiol. 87, 946 (2011).

6. Gibson, E. A. Dye-sensitized photocathodes for $\mathrm{H}_{2}$ evolution. Chem. Soc. Rev. 46, 6194 (2017).

7. Freys, J. C., Gardner, J. M., D’Amario, L., Brown, A. M. \& Hammarström, L. Ru-based donor-acceptor photosensitizer that retards charge recombination in a p-type dye-sensitized solar cell. Dalton Trans. 41, 13105 (2012).

8. Lefebvre, J.-F. et al. An artificial photosynthetic system for photoaccumulation of two electrons on a fused dipyridophenazine (dppz)-pyridoquinolinone ligand. Chem. Sci. 9, 4152 (2018).

9. Queyriaux, N. et al. Aqueous photocurrent measurements correlated to ultrafast electron transfer dynamics at ruthenium tris diimine sensitized NiO photocathodes. J. Phys. Chem. C. 121, 5891 (2017).

10. Massin, J. et al. Dye-sensitized PS-b-P2VP-templated nickel oxide films for photoelectrochemical applications. Interface Focus. 5, $20140083(2015)$

11. Karlsson, S. et al. Accumulative electron transfer: Multiple charge separation in artificial photosynthesis. Faraday Discuss. 155, 233 (2012).

12. Hammarström, L. Accumulative charge separation for solar fuels production: Coupling light-induced single electron transfer to multielectron catalysis. Acc. Chem. Res. 48, 840 (2015).

13. Wood, C. J. et al. A comprehensive comparison of dye-sensitized NiO photocathodes for solar energy conversion. Phys. Chem. Chem. Phys. 18, 10727-10738 (2016).

14. Zhang, F., Johansson, M., Andersson, M. R., Hummelen, J. C. \& Inganäs, O. Polymer photovoltaic cells with conducting polymer anodes. Adv. Mater. 14, 662 (2002).

15. Chang, E. C., Chao, C.-I. \& Lee, R.-H. Enhancing the efficiency of MEH-PPV and PCBM based polymer solar cells via optimization of device configuration and processing conditions. J. Appl. Polym. Sci. 101, 1919 (2006).

16. Facchetti, A. Polymer donor-polymer acceptor (all-polymer) solar cells. Mater. Today. 16, 123-132 (2013).

17. Suzuki, M. et al. Hydrogen generation using water-insoluble polymer-bound ruthenium(II) complexes. Chem. Commun. 2, 227-228 (1997).

18. Suzuki, M., Kimura, M., Hanabusa, K. \& Shirai, H. Photoinduced hydrogen generation using polymer photosensitizers. Macromol. Chem. Phys. 199, 945 (1998).

19. Suzuki, M. et al. Photoinduced hydrogen generation from water-insoluble polymer photosensitizer films. Polymer 39, 1539 (1998).

20. Suzuki, M., Kimura, M., Hanabusa, K. \& Shirai, H. Intra-polymer photosensitized charge separation using a series of ruthenium(II) complex and viologen-containing polymers. Acta Polym. 50, 45 (1999).

21. Jiang, J. et al. Polymer chromophore-catalyst assembly for solar fuel generation. ACS Appl. Mater. Interfaces. 9, 19529 (2017).

22. Leem, G. et al. Ru(bpy) ${ }_{3}{ }^{2+}$ derivated polystyrenes constructed by nitroxide-mediated radical polymerization. Relationship between polymer chain length, structures and photophysical properties. Polym. Chem. 6, 8184 (2015).

23. Leem, G., Sherman, B. D. \& Schanze, K. S. Polymer-based chromophore-catalyst assemblies for solar energy conversion. Nano Converg. 4, 37 (2017).

24. Dupray, L. M. \& Meyer, T. J. Synthesis and characterization of amide-derivated, polypyridyl-based metallopolymers. Inorg. Chem. 35, 6299 (1996).

25. Fang, Z. et al. Atom transfer radical polymerization preparation and photophysical properties of polypyridylruthenium derivatized polystyrenes. Inorg. Chem. 52, 8511 (2013).

26. Peng, Z. \& Yu, L. Synthesis of conjugated polymers containing ionic transition metal complexes. J. Am. Chem. Soc. 1187, 3777 (1996).

27. Wang, Q., Wang, L. \& Yu, L. Synthesis and unusual physical behavior of a photorefractive polymer containing tris(bipyridyl) ruthenium(II) complexes as a photosensitizer and exhibiting a low glass-transition temperature. J. Am. Chem. Soc. 120, 12860 (1998).

28. Wang, Q. \& Yu, L. Conjugated polymer containing mixed-ligand ruthenium(II) complexes. Synthesis, characterization, and investigation of photoconductive properties. J. Am. Chem. Soc. 122, 11806 (2000).

29. Sariciftci, N. S., Smilowitz, L., Heeger, A. J. \& Wudl, F. Photoinduced electron transfer from a conducting polymer to buckminsterfullerene. Science 258, 1474 (1992).

30. Shaheen, S. E., Brabec, C. J. \& Sariciftci, N. S. 2.5\% efficient organic plastic solar cells. Appl. Phys. Lett. 78, 841 (2001).

31. Greenwald, Y. et al. Polymer-polymer rectifying heterojunction based on poly(3,4-dicyanothiophene) and MEH-PPV. J. Pol. Sci. 36, 3115 (1998). 
32. Xiao, Y., Yu, W.-L., Chua, S.-J. \& Huang, W. A novel series of copolymer containing 2,5-Dicyano-1,4-phenylene-vinylene-Synthetic tuning of the HOMO and LUMO energy levels of conjugated polymers. Chem. Eur. J. 6, 1318 (2000).

33. Irwin, M. D., Buchholz, D. B., Hains, A. W., Chang, R. P. H. \& Marks, T. J. p-Type semiconducting nickel oxide as an efficiencyenhancing anode interfacial layer in polymer bulk-heterojunction solar cells. Proc. Nalt. Acad. Sci. 105, 2783 (2008).

34. Stevanovic, V., Lany, S., Ginley, D. S., Tumas, W. \& Zunger, A. Assessing capability of semiconductors to split water using ionization potentials and electron affinities only. Phys. Chem. Chem. Phys. 15, 3706 (2014).

35. Wintergerst, P., Mengele, A. K., Nauroozi, D., Tschierlei, S. \& Rau, S. Impact of alkyne functionalization on photophysical and electrochemical properties of 1,10-phenanthrolines and their $\mathrm{Ru}^{\mathrm{II}}$ complexes. Eur. J. Inorg. Chem. 2019, 1988-1992 (2019).

36. Sumpter, B. G. et al. Computational study of the structure, dynamics, and photophysical properties of conjugated polymers and oligomers under nanoscale confinement. J. Phys. Chem. B. 109, 7671 (2005).

37. Correia, H. M. G. \& Ramos, M. M. D. Modelling the effect of nonplanarity on charge transport along conjugated polymer chains. Mater. Sci. Eng. C. 27, 1133 (2007).

38. Milad, R. et al. Effective conjugation in conjugated polymers with strongly twisted backbones: A case study on fluorinated MEHPPV. J. Mater. Chem. C. 4, 6900 (2016).

39. Liu, L. T., Yaron, D. \& Berg, M. A. Electron-phonon coupling in phenyleneethynylene oligomers: A nonlinear one-dimensional configuration-coordinate model. J. Phys. Chem. C 111, 5770 (2007).

40. Bazan, G. C., Miao, Y.-J., Renak, M. L. \& Sun, B. J. Fluorescence quantum yield of poly(p-phenylenevinylene) prepared yia the paracyclophene route: Effect of chain length and interchain contacts. J. Am. Chem. Soc. 118, 2618 (1996).

41. Brown, A. R. et al. Poly(p-phenylenevinylene) light-emitting diodes: Enhanced electroluminescent efficiency through charge carrier confinement. Appl. Phys. Lett. 61, 2793 (1992).

42. Hwang, I. \& Scholes, G. D. Electronic energy transfer and quantum-coherence in $\pi$-conjugated polymers. Chem. Mater. 23, 610 (2011).

43. Dykstra, T. E. et al. Conformational disorder and ultrafast exciton relaxation in PPV-family conjugated polymers. J. Phys. Chem. B. 113, 656 (2009)

44. Sun, Y. et al. Light harvesting arrays of polypyridine ruthenium(II) chromophores prepared by Reversible Addition-Fragmentation Chain Transfer polymerization. Macromolecules 45, 2632 (2012).

45. Bräutigam, M., Kübel, J., Schulz, M., Vos, J. G. \& Dietzek, B. Hole injection dynamics from two structurally related Ru-bipyridine complexes into NiOx is determined by the substitution pattern of the ligands. Phys. Chem. Chem. Phys. 17, 7823-7830 (2015).

46. Damrauer, N. H. et al. Femtosecond dynamics of excited-state evolution in $\left[\mathrm{Ru}(\mathrm{bpy})_{3}\right]^{2+}$. Science 275, 54-57 (1997).

47. Damrauer, N. H. \& McCusker, J. K. Ultrafast dynamics in the metal-to-ligand charge transfer excited state evolution of $\left[\mathrm{Ru}\left(4,4^{\prime}-\right.\right.$ diphenyl-2,2'-bipyridine) $]_{3}^{2+}$. J. Phys. Chem. A. 103, 8440 (1999).

48. Brouwer, A. M. Standards for photoluminescence quantum yield measurements in solution (IUPAC Technical report). Pure Appl. Chem. 83, 2213 (2011).

49. Vandewal, K., Benduhn, J. \& Nikolis, V. C. How to determine optical gaps and voltage losses in organic photovoltaic materials. Sustain. Energy Fuels. 2, 538 (2018).

50. Meskers, S. C. J., Janssen, R. A. J., Haverkort, J. E. M. \& Wolter, J. H. Relaxation of photo-excitations in films of oligo- and poly(para-phenylene vinylene) derivatives. Chem. Phys. 260, 415 (2000).

51. Herz, L. M., Silva, C., Grimsdale, A. C., Müllen, K. \& Phillips, R. T. Time-dependent energy transfer rates in a conjugated polymer guest-host system. Phys. Rev. B. 70, 165207 (2004).

52. Nguyen, T.-Q., Wu, J., Doan, V., Schwartz, B. J. \& Tolbert, S. H. Control of energy transfer in oriented conjugated polymermesoporous silica composites. Science 288, 652 (2000).

53. Grage, M.M.-L. et al. Conformational disorder and energy migration in MEH-PPV with partially broken conjugation. J. Chem. Phys. 118, 7644 (2003).

54. Gaab, K. M. \& Bardeen, C. J. Anomalous exciton diffusion in the conjugated polymer MEH-PPV measured using a three-pulse pump-dump-probe anisotropy experiment. J. Phys. Chem. A. 108, 10801 (2004).

55. D’Amario, L., Antila, L. J., Rimgard, B. P., Boschloo, G. \& Hammarström, L. Kinetic evidence of two pathways for charge recombination in NiO-based dye-sensitized solar cells. J. Phys. Chem. Lett. 6, 779 (2015).

56. Han, Y. et al. Interfacial electron transfer yields in dye-sensitized $\mathrm{NiO}$ photocathodes correlated to excited-state dipole orientation of ruthenium chromophores. Can. J. Chem. 96, 865 (2017).

57. Dillon, R. J., Alibabaei, L., Meyer, T. J. \& Papanikolas, J. M. Enabling efficient creation of long-lived charge-separation on dyesensitized $\mathrm{NiO}$ photocathodes. ACS Appl. Mater. Interfaces. 9, 26786 (2017).

58. Wang, L. et al. Competition between ultrafast energy flow and electron transfer in a Ru(II)-Loaded polyfluorene light-harvesting polymer. J. Phys. Chem. Lett. 3, 2453-2457 (2012).

59. Leem, G. et al. Light harvesting and charge separation in a $\pi$-conjugated antenna polymer bound to $\mathrm{TiO}_{2}$. J. Phys. Chem. C. 118, 28535-28541 (2014)

60. Ley, K. D., Whittle, C. E., Bartberger, M. D. \& Schanze, K. S. Photophysics of $\pi$-conjugated polymers that incorporate metal to ligand charge transfer chromophores. J. Am. Chem. Soc. 119, 3423-3424 (1997).

61. Duprez, V., Biancardo, M., Spanggaard, H. \& Krebs, F. C. Synthesis of conjugated polymers containing terpyridine-ruthenium complexes: Photovoltaic applications. Macromolecules 38, 10436-10448 (2005).

62. Morgado, J. et al. Föster energy transfer and control of the luminescence in blends of an orange-emitting poly(p-phenylenevinylene) and a red-emitting tetraphenylporphyrin. J. Mater. Chem. 11, 278-283 (2001).

63. Burrows, H. D., da Miguel, M. G., Monkman, A. P., Hamblett, I. \& Navaratnam, S. Transient absorption spectra of triplet states and charge carriers of conjugated polymer. J. Mol. Struct. 563-564, 41-50 (2001).

64. Monkman, A. P., Burrows, H. D., da Miguel, M. G., Hamblett, I. \& Navaratnam, S. Triplet state spectroscopy of conjugated polymers studied by pulse radiolysis. Synth. Met. 116, 75-79 (2001).

65. Markov, D. E., Tanase, C., Blom, P. W. M. \& Wildeman, J. Simultaneous enhancement of charge transport and exciton diffusion in poly(p-phenylene vinylene) derivatives. Phys. Rev. B. 72, 045217 (2005).

66. Rörich, I., Mikhnenko, O. V., Gehrig, D., Blom, P. W. M. \& Gracium, N. I. Influence of energetic disorder on exciton lifetime and photoluminescence efficiency in conjugated polymers. J. Phys. Chem. B. 121, 1405 (2017).

67. Atreya, M. et al. Stability studies of poly(2-methoxy-5-(2'-ethyl hexyloxy)-p-(phenylene vinylene) [MEH-PPV]. Polym. Degrad. Stab. 65, 287 (1999).

68. Lim, Y. T., Lee, T.-W., Lee, H.-C. \& Park, O. O. Enhanced photo-stability of conjugated polymer nanocomposites doped with functionalized nanoparticles. Opt. Mater. 21, 585 (2002).

69. Lyu, $\mathrm{S}$. et al. $\mathrm{H}_{2}$-evolving dye-sensitized photocathode based on a ruthenium-diacetylide/cobaloxime supramolecular assembly. ACS Appl. Energy Mater. 2, 4971-4980 (2019).

70. Massin, J. et al. Investigating light-driven hole injection and hydrogen evolution catalysis at dye-sensitized $\mathrm{NiO}$ photocathodes: A combined experimental-theoretical study. J. Phys. Chem. C 123, 17176-17184 (2019).

71. Schroot, R., Schlotthauer, T., Dietzek, B., Jäger, M. \& Schubert, U. S. Extending long-lived charge separation between donor and acceptor blocks in novel copolymer architectures featuring a sensitizer core. Chem. Eur. J. 23, 16484 (2017).

72. Schindler, J. et al. A $\pi \pi^{*}$ states enables photoaccumulation of charges on a $\pi$-extended dipyridophenazine ligand in a $\mathrm{Ru}(\mathrm{II})$ polypyridine complex. J. Phys. Chem. C 122, 83-95 (2018). 
73. Zhang, Y. et al. In situ spectroelectrochemical and theoretical study on the oxidation of a $4 \mathrm{H}$-imidazole-ruthenium dye adsorbed on nanocrystalline $\mathrm{TiO}_{2}$ thin film electrodes. Phys. Chem. Phys. Chem. 17, 29637 (2015).

74. Zedler, L. et al. Unraveling the light-activated reaction mechanism in a catalytically competent key intermediate of multifunctional molecular catalyst for artificial photosynthesis. Angew. Chem. Int. Ed. 58, 13140-13148 (2019).

75. Monczak, K. et al. Synthesis and characterization of a trisheteroleptic RuII-based molecular switch. Chem. Eur. J. 20, 15426-15433 (2014).

76. Wächtler, M. et al. Structural control of photoinduced dynamics in 4 H-imidazole-ruthenium dyes. J. Phys. Chem. C. 116, 2566425676 (2012).

77. Bräutigam, M., Wächtler, M., Rau, S., Popp, J. \& Dietzek, B. Photophysical dynamics of a ruthenium polypyridine dye controlled by solvent pH. J. Phys. Chem. C. 116, 1274-1281 (2012).

78. Dietzek, B., Pascher, T., Sundström, V. \& Yartsev, A. Appearance of coherent artifact signals in femtosecond transient absorption spectroscopy in dependence on detector design. Laser Phys. Lett. 4, 38 (2007).

79. Luo, Y. et al. Energy versus electron transfer: Controlling the excitation transfer in molecular triads. Chem. Eur. J. 23, 4917 (2017).

80. Barthelmes, K. et al. New ruthenium Bis(terpyridine) methanofullerene and pyrrolidinofullerene complexes: Synthesis and electrochemical and photophysical properties. Inorg. Chem. 54, 3159 (2015).

\title{
Acknowledgements
}

This work was funded by the Deutsche Forschungsgemeinschaft (DFG, German Research Foundation)—project number 364549901-TRR 234 [CataLight Project C1, B2 and B4]. Financial support by the Deutscher Akademischer Austauschdienst (DAAD) for PhD Scholarship for RAW is gratefully acknowledged. SB acknowledges support by the French National Research Agency in the framework of the "Investissements d'avenir" program (ANR-15-IDEX-02 and CBH-EUR-GS, ANR-17-EURE-0003).

\section{Author contributions}

B.D., S.R., U.S.S., M.D.H., C.S. M.W. conceived the project, J.H. and P.W. synthesized and characterized PPV, Ru, and PPV-Ru, R.A.W. carried out fs-transient absorption measurements, R.A.W. and S.B. carried out nstransient absorption measurements, R.A.W., B.S., and G.L. performed electrochemistry and spectroelectrochemistry measurements, A.D. and J.D. carried out SEM measurement and analysis, B.S. and S.B. carried out photoelectrochemical measurements, R.A.W, B.D., and M.W. analysed the data, R.A.W. and B.D. wrote the initial manuscript. All authors discussed the results and contribute to the final manuscript.

\section{Funding}

Open Access funding enabled and organized by Projekt DEAL.

\section{Competing interests}

The authors declare no competing interests.

\section{Additional information}

Supplementary Information The online version contains supplementary material available at https://doi. org/10.1038/s41598-021-82395-x.

Correspondence and requests for materials should be addressed to B.D.

Reprints and permissions information is available at www.nature.com/reprints.

Publisher's note Springer Nature remains neutral with regard to jurisdictional claims in published maps and institutional affiliations.

\begin{abstract}
Open Access This article is licensed under a Creative Commons Attribution 4.0 International License, which permits use, sharing, adaptation, distribution and reproduction in any medium or format, as long as you give appropriate credit to the original author(s) and the source, provide a link to the Creative Commons licence, and indicate if changes were made. The images or other third party material in this article are included in the article's Creative Commons licence, unless indicated otherwise in a credit line to the material. If material is not included in the article's Creative Commons licence and your intended use is not permitted by statutory regulation or exceeds the permitted use, you will need to obtain permission directly from the copyright holder. To view a copy of this licence, visit http://creativecommons.org/licenses/by/4.0/.
\end{abstract}

(c) The Author(s) 2021 